\title{
Comparing the effects of acute alcohol consumption in germ-free and conventional mice: the role of the gut microbiota
}

Canesso MCC ${ }^{1,5}$, Lacerda $\mathrm{NL}^{2}$, Ferreira $\mathrm{CM}^{2,6}$, Gonçalves $\mathrm{JL}^{2}$, Almeida D², Gamba C ${ }^{3}$, Cassali G ${ }^{3}$, Pedroso SH ${ }^{4}$, Moreira $\mathrm{C}^{4}$, Martins $\mathrm{FS}^{4}$, Nicoli $\mathrm{JR}^{4}$, Teixeira $\mathrm{MM}^{5}$, Godard $\mathrm{ALB}^{2^{*}}$ and Vieira $\mathrm{AT}^{2,4,5^{*}}$

\begin{abstract}
Background: Increasing evidence suggest that the gut microbiota plays an important role in liver pathology after acute alcohol intake. The aim of our study was to investigate the roles played by commensal bacteria in alcohol-induced liver injury and in the dysbiosis caused by alcohol intake in germ-free mice, as well as the possibility of protection against alcohol-induced injuries in animals fed a high-fiber diet. For these purposes, germ-free and conventional mice were submitted to acute alcohol intake, consisting of administration of ethanol in their drinking water for 7 days, with a higher dose of alcohol administered on day 7 .

Results: There was no liver injury after alcohol consumption, and there was less neutrophil infiltration and lower pro-inflammatory cytokine levels (CXCL-1/KC and interleukin (IL)-6) in the liver in germ-free mice compared with alcohol-fed conventional mice. Additionally, conventionalization of germ-free mice with intestinal contents from alcohol-fed conventional mice induced injury and inflammation in both the liver and the intestine, suggesting that alcohol intake successively caused a perturbation of the intestinal microbiota (dysbiosis) and liver injury. Finally, previous treatment with a high-fiber diet decreased liver injury and gut permeability in alcohol-fed conventional mice.

Conclusions: In conclusion, the results of the present study provide evidence that the gut microbiota plays an important role in alcohol-induced liver injury, apparently through dysbiosis of the intestinal microbial ecosystem caused by alcohol intake. Furthermore, treatment with a high-fiber diet can counteract hepatocyte pathology and gut leakage and thus could be a promising therapeutic option.
\end{abstract}

Keywords: Alcohol, Germ-free, Microbiota, Dysbiosis, Fiber, Liver, Gut, Inflammation

\section{Background}

Alcohol consumption, which is associated with severe hepatic damage (e.g., fibrosis and cirrhosis) [1,2], is one of the major causes of chronic liver disease in Western countries [3]. Of the many factors that contribute to the pathogenesis of alcohol-induced liver injury, gut-derived bacterial products seem to play a central role in inducing steatosis and inflammation. In particular, a high level of lipopolysaccharide (LPS) is found in the blood of patients

\footnotetext{
* Correspondence: brunialt@icb.ufmg.br; angelicathomaz@gmail.com

${ }^{2}$ Department of General Biology, Institute of Biological Sciences, Universidade

Federal de Minas Gerais, Belo Horizonte, Brazil

${ }^{4}$ Department of Microbiology, Institute of Biological Sciences, Universidade

Federal de Minas Gerais, Belo Horizonte, Brazil

Full list of author information is available at the end of the article
}

with chronic alcohol consumption, and this phenomenon is related to a range of factors, including a predominant change in the composition of the intestinal microbiota $[4,5]$. This endotoxin may favor alteration of the balance of normal bacteria, hindrance of beneficial commensal bacteria, increase intestinal permeability by producing inflammatory mediators and alcohol metabolites in the intestine, and contribute to dysfunction of the intestinal epithelial cells and alteration of tight junctions [6]. It has been observed that increased intestinal permeability facilitates the translocation of microbial products, such as LPS, from the intestinal lumen to the extra-intestinal organs [7]. After these gut-derived bacterial products 
translocate from the gut to the liver, they interact with TLR4 on Küpffer cells, leading to production of TNF- $\alpha$ and oxidative stress, which cause hepatocellular damage [3].

Another factor that contributes to the severity of alcoholic liver disease (ALD) is the infiltration of the liver by neutrophils. Studies have shown that the systemic activation of neutrophils by pro-inflammatory cytokines, chemokines, complement factors, and other biologically active molecules (e.g., platelet-activating factor) can lead to the migration of neutrophils to the liver, where they kill sensitized hepatocytes by releasing inflammatory mediators [8].

The intestinal microbiota has emerged as an important factor in triggering inflammatory responses. Because products of the unbalanced normal intestinal microbiota are important mediators of the hepatic injury induced by excessive alcohol intake, alterations in the composition of the intestinal microbiota may explain its role in the pathogenesis of liver injury. In fact, recent studies have confirmed that the intestinal microbial composition is altered in alcoholism [9,10]. This alteration (dysbiosis) could be one of the factors that may exacerbate inflammatory responses, even systemically, after alcohol ingestion. Thus, restoring the normal composition of the intestinal microbiota may be an alternative treatment for reducing the hepatic injury caused by excessive alcohol intake. Probiotic, prebiotic and high-fiber diets are several of the possible ways to intervene in the intestinal microbial ecosystem.
In the present study, it was hypothesized that alcohol consumption alters the composition of the gut microbiota, causing dysbiosis, and that this alteration plays a role in the inflammatory response induced in the liver. To investigate this hypothetical role of the microbiota, germ-free mice were compared with their conventional counterparts in an acute alcohol intake model. In addition, the treatment of mice with a high-fiber diet was evaluated as a possible means to decreasing dysbiosis of the microbiota and the consequent liver injury.

\section{Results}

Germ-free mice show reduced liver pathology after alcohol administration compared with conventional mice Lipid accumulation in the liver is an important characteristic of hepatic pathology that is known as steatosis. To evaluate hepatocyte damage in the mouse groups, we measured the lipid levels in the liver. We observed that germ-free mice that were subjected to alcohol treatment showed no altered liver lipid content nine hours after the high dose of alcohol was administered on day 7. In contrast, the livers of the conventional mice showed increased lipid levels $(\mathrm{p}<0.01)$ at the same time (Figure $1 \mathrm{~A})$. Moreover, germ-free mice treated with alcohol did not show alteration in gut permeability compared with control germ-free mice. In contrast, conventional mice increased intestinal permeability after excessive alcoholic intake (Figure 1B). Score of liver damage histopathology confirmed that ethanol consumption induced the formation

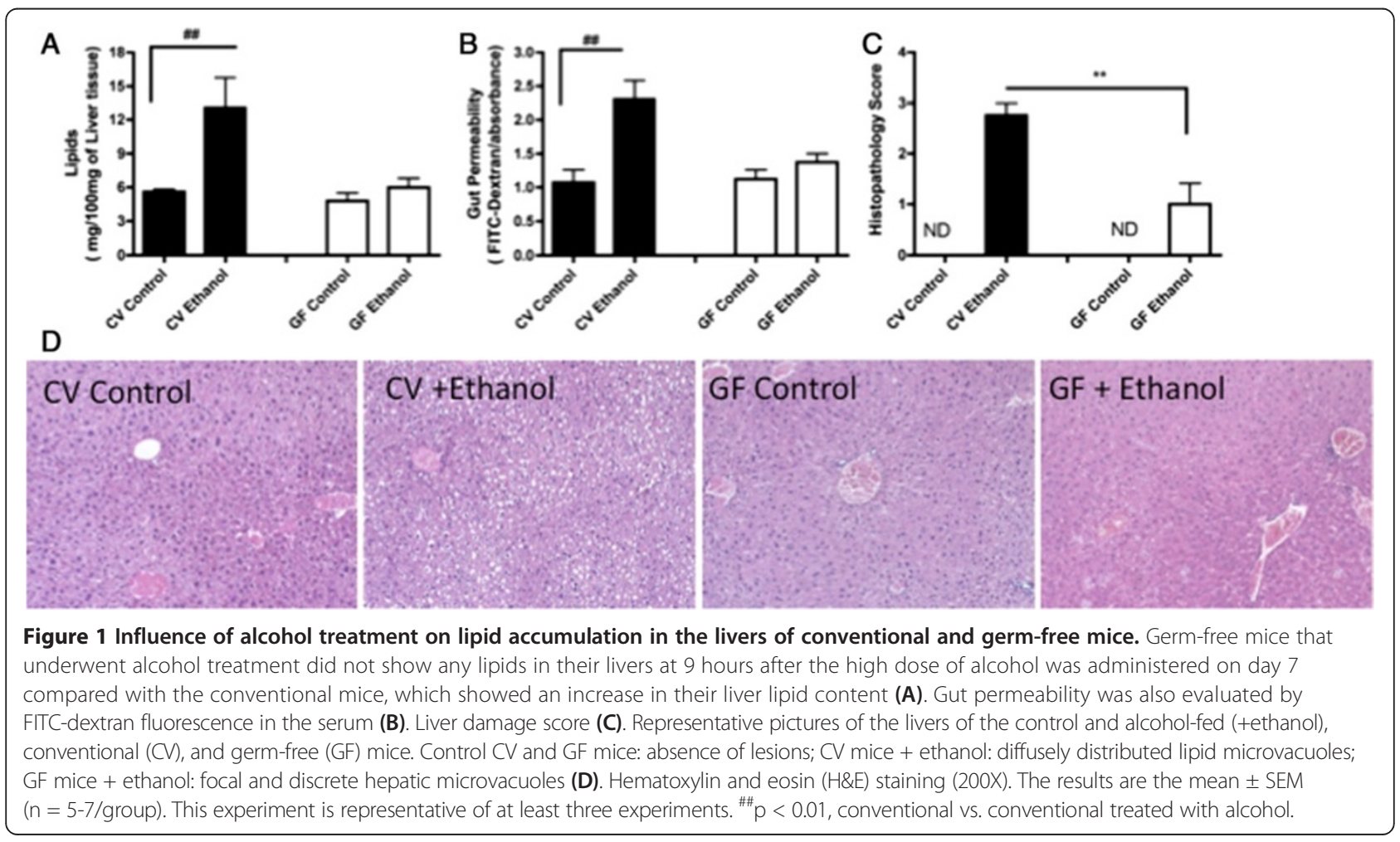


of lipid microvacuoles that were diffusely distributed in the conventional mice $(\mathrm{CV}+$ Ethanol) and focally distributed in the germ-free mice (GF + Ethanol) (Figure 1C, D). There was no difference in hydric consumption between the groups tested (Additional file 1).

The profiles of the inflammatory response in the livers of conventional and germ-free mice were also examined. Neutrophils are important inflammatory cells that contribute to ALD, as they infiltrate the liver after being activated by pro-inflammatory cytokines and kill sensitized hepatocytes [8]. To evaluate neutrophil accumulation in the liver, we used a myeloperoxidase (MPO)-based activity assay. MPO is the most abundant enzyme in neutrophils, and it has been shown to be a useful and reliable marker for neutrophil infiltration [11]. We observed an increased level of neutrophil infiltration in the livers of the conventional mice $(\mathrm{p}<0.05)$ after the final high dose of alcohol was administered on day 7 compared with the conventional control group (Figure 2A). In contrast, no difference was observed in the neutrophil accumulation in the livers of the germ-free mice after alcohol treatment (Figure 2A). In agreement with the liver lipid and neutrophil data, alcohol intake also increased the levels of the pro-inflammatory cytokines CXCL-1/KC and interleukin (IL) -6 in the livers of the conventional mice ( $\mathrm{p}<0.05$ and $\mathrm{p}<0.01$, respectively) but did not affect these levels in the germ-free mouse livers (Figures 2B-C). Together, these findings indicate that the indigenous microbiota plays an important role in hepatocyte pathology after alcohol consumption.

\section{Alcohol consumption causes intestinal bacterial overgrowth and dysbiosis}

Given that the inflammatory response after alcohol intake was more prominent in the small intestine (data not shown), we sought to determine whether alcohol intake leads to intestinal dysbiosis. Therefore, we collected the contents of the small intestines of control conventional mice and conventional mice treated with alcohol to quantify the CFUs in different media. Our results showed that alcohol consumption increased the number of cultivable bacteria observed, based on the CFUs counted, and thus increased the total number of bacteria compared with the numbers in the control mice $(\mathrm{p}<0.05)$ (Table 1). Interestingly, the largest difference between the groups was observed in the numbers of enterobacteria $(\mathrm{p}<0.001)$.
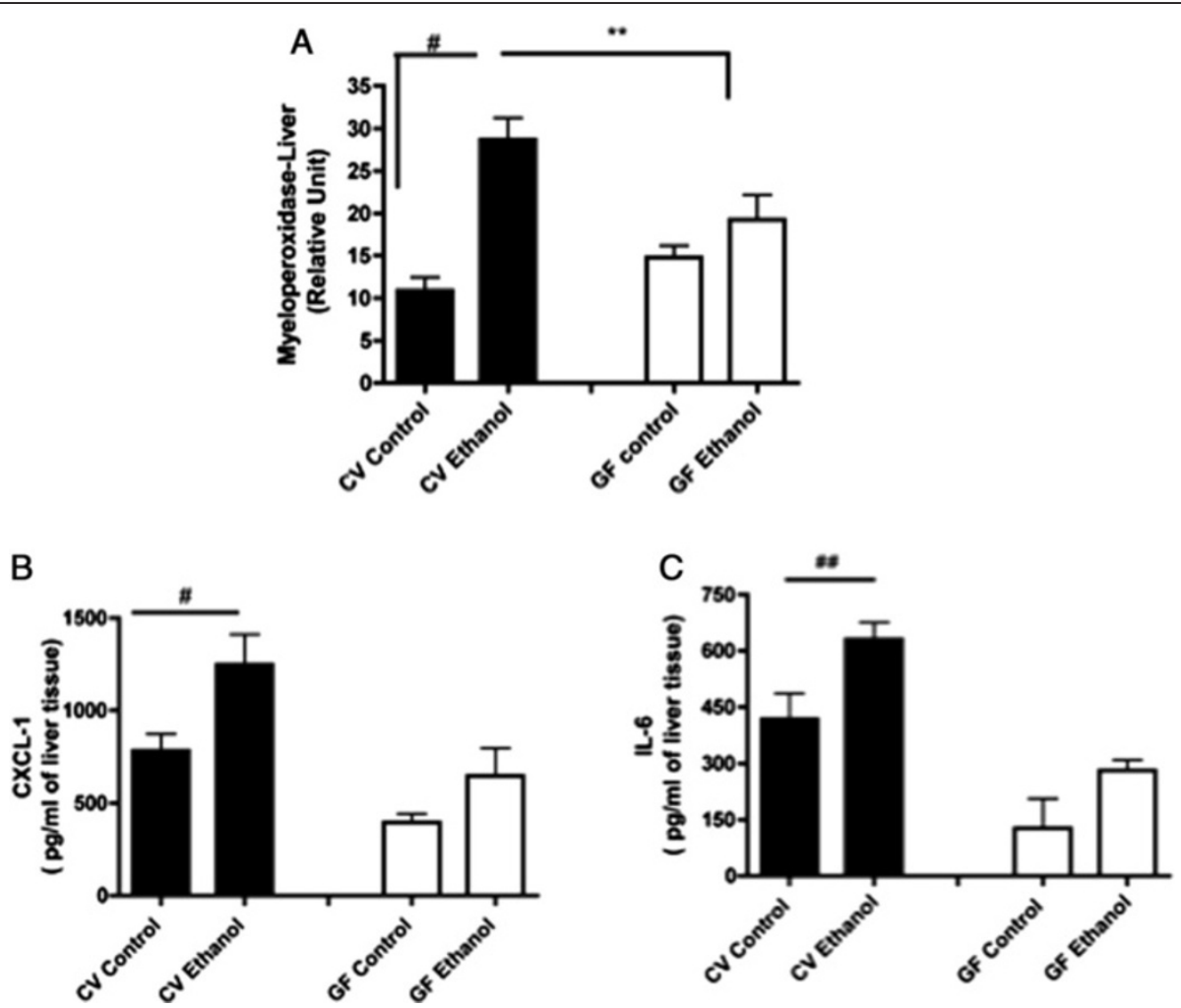

Figure 2 Influence of alcohol treatment on inflammatory parameters in the livers of germ-free and conventional mice. The number of neutrophils that had accumulated in the liver was estimated using an MPO assay (A). Levels of the pro-inflammatory cytokines CXCL-1/KC and IL-6 in the liver (B and C, respectively). The cytokine levels were measured by ELISA. The data represent the mean \pm SEM ( $n=5-7 / g r o u p)$. ${ }^{\#} p<0.05,{ }^{\# \#} p<0.01,9$ hours vs. 0 hours after the high dose of alcohol was administered; ${ }^{* *} p<0.01$, conventional vs. germ-free mice. 
Table 1 Quantitative analysis of microbial populations in conventional mice treated with or without alcohol utilizing the feces cultivation-dependent method (CFU/g of feces)

\begin{tabular}{llll}
\hline Selected media & Selected microorganisms & \multicolumn{2}{l}{ Experimental groups } \\
\cline { 2 - 4 } & & Control & Ethanol \\
\hline MacConkey & Enterobacteria & $1.7 \times 10^{8} \pm 1745$ & $1.9 \times 10^{9} \pm 1500\left(^{* * *}\right)$ \\
Heart Infusion Broth with Azide & Enterococcus & $1.0 \times 10^{5} \pm 0.68$ & $4.0 \times 10^{8} \pm 3986\left(^{*}\right)$ \\
Supplemented Blood Media (Cultivated in $\mathbf{O}_{2}$ ) & Total Aerobic Bacteria & $5.6 \times 10^{8} \pm 2534$ & $2.6 \times 10^{9} \pm 6699\left(^{*}\right)$ \\
Supplemented Blood Media (Cultivated in $\left.\mathbf{C O}_{2}\right)$ & Total Anaerobic Bacteria & $6.4 \times 10^{8} \pm 3640$ & $2.1 \times 10^{9} \pm 3987\left(^{*}\right)$ \\
MRS & Lactic Acid Bacteria & $4.9 \times 10^{8} \pm 2593$ & $2.7 \times 10^{9} \pm 6071\left(^{*}\right)$ \\
\hline
\end{tabular}

(The data represent the mean \pm SEM, $\left.{ }^{*} \mathrm{p}<0.05 ; * * * \mathrm{p}<0.001\right)$.

Conventionalization of germ-free mice with intestinal contents from alcohol-fed conventional mice induces inflammation in the small intestine and the liver

To determine whether the commensal microbiota or a dysbiotic alcohol-altered microbiota is important to liver injury, germ-free mice were administered the intestinal contents of other germ-free mice (GF $\rightarrow$ GF) or were conventionalized with intestinal contents from conventional mice treated with $(\mathrm{CV}+\mathrm{Eth} \rightarrow \mathrm{GF})$ or without $(\mathrm{CV} \rightarrow \mathrm{GF})$ alcohol. These animals then underwent the same alcohol treatment as described above. In the $\mathrm{CV} \rightarrow \mathrm{GF}$ group, compared with the GF $\rightarrow$ GF group, increases in neutrophil accumulation (Figure $3 \mathrm{~A}$ ) and in the levels of the pro-inflammatory cytokines CXCL-1/KC (Figure 3B) and IL-6 (Figure 3C) were observed in the liver after treatment with alcohol. Interestingly, these increases were even more pronounced in the $\mathrm{CV}+\mathrm{Eth} \rightarrow$ GF group compared with the $\mathrm{CV} \rightarrow$ GF group. Histopathological score evaluation confirmed these data in both $\mathrm{CV} \rightarrow \mathrm{GF}$ and $\mathrm{CV}+\mathrm{Eth} \rightarrow \mathrm{GF}$ mice, showing increased multifocal cytoplasmic microvacuolation than in ethanoltreated GF $\rightarrow$ GF mice (Figure 3D, E).

The greater effect of the dysbiotic, alcohol-altered microbiota on liver injury observed above was further observed at the small intestinal level, as demonstrated by the higher values obtained for the clinical score, neutrophil accumulation and CXCL-1/KC levels in $\mathrm{CV}+$ Eth $\rightarrow$ GF mice compared with GF $\rightarrow$ GF and $\mathrm{CV} \rightarrow$ GF mice (Figures $4 \mathrm{~A}, 4 \mathrm{~B}$ and $4 \mathrm{C}$, respectively). Altogether, these data suggest that excessive alcohol intake leads to a dysbiotic microbiota, which is crucial in promoting inflammation, gut leakage, and liver injury [9].

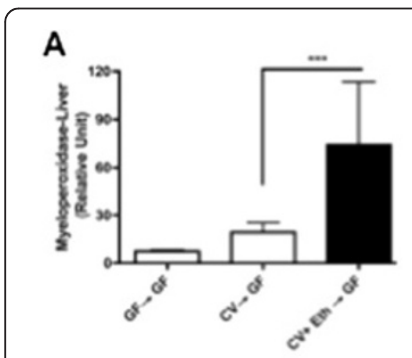

E
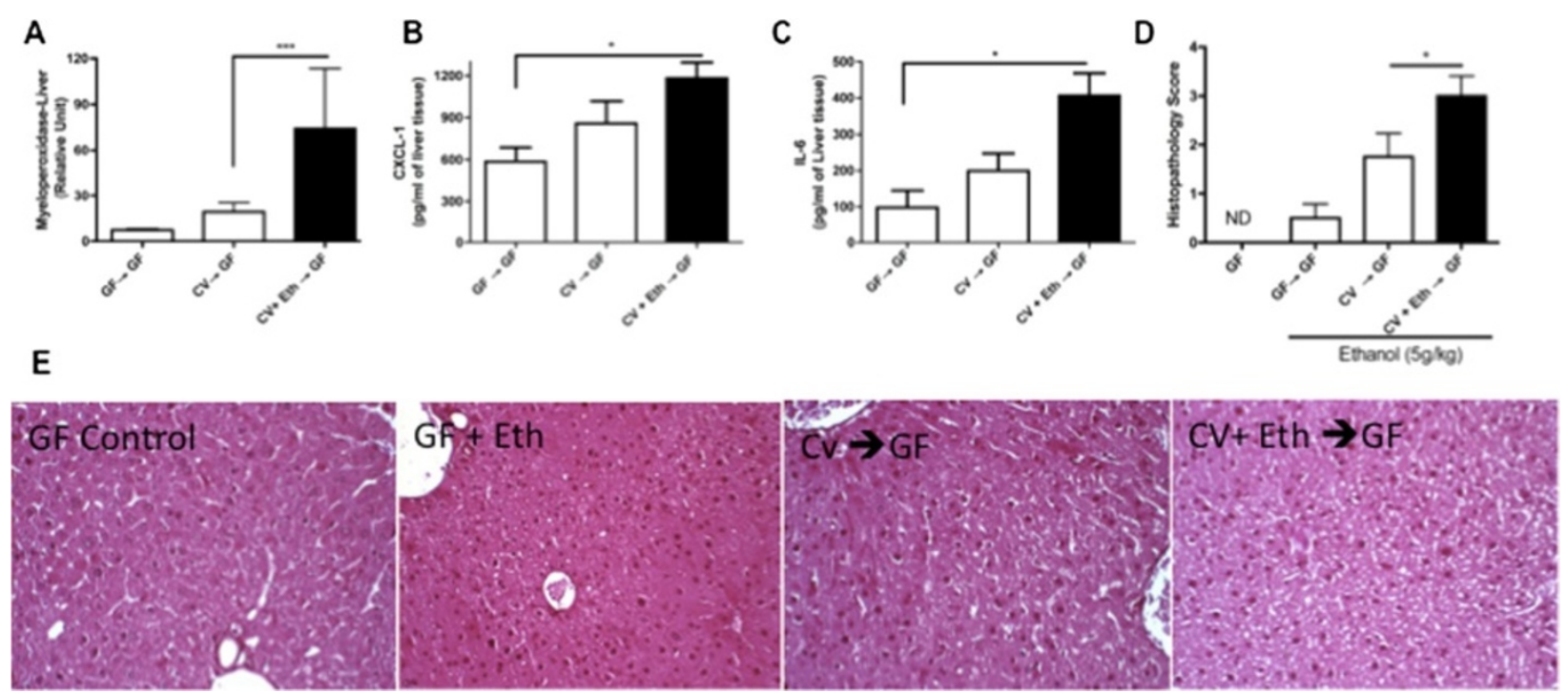

Figure 3 Influence of alcohol treatment on inflammatory parameters in the livers of germ-free mice conventionalized with intestinal contents from conventional mice. The number of neutrophils that had accumulated in the liver was measured using an MPO assay (A). Proinflammatory cytokine (CXCL-1/KC [B] and IL-6 [C]) levels in the liver. The cytokine levels were measured using ELISA. Liver score (D). Representative pictures of the livers of the germ-free mice (GF Control), the germ-free mice that underwent alcohol treatment (GF + Ethanol), the germ-free mice colonized with intestinal contents from the conventional mice ( $C V \rightarrow G F)$, and the germ-free mice colonized with intestinal contents from the conventional mice treated with alcohol (CV + Eth $\rightarrow$ GF) (E). PAS staining (200X). The data represent the mean \pm SEM $(n=5-7 / g r o u p)$. ${ }^{*} \mathrm{p}<0.05 ;{ }^{* * *} \mathrm{p}<0.001$. 

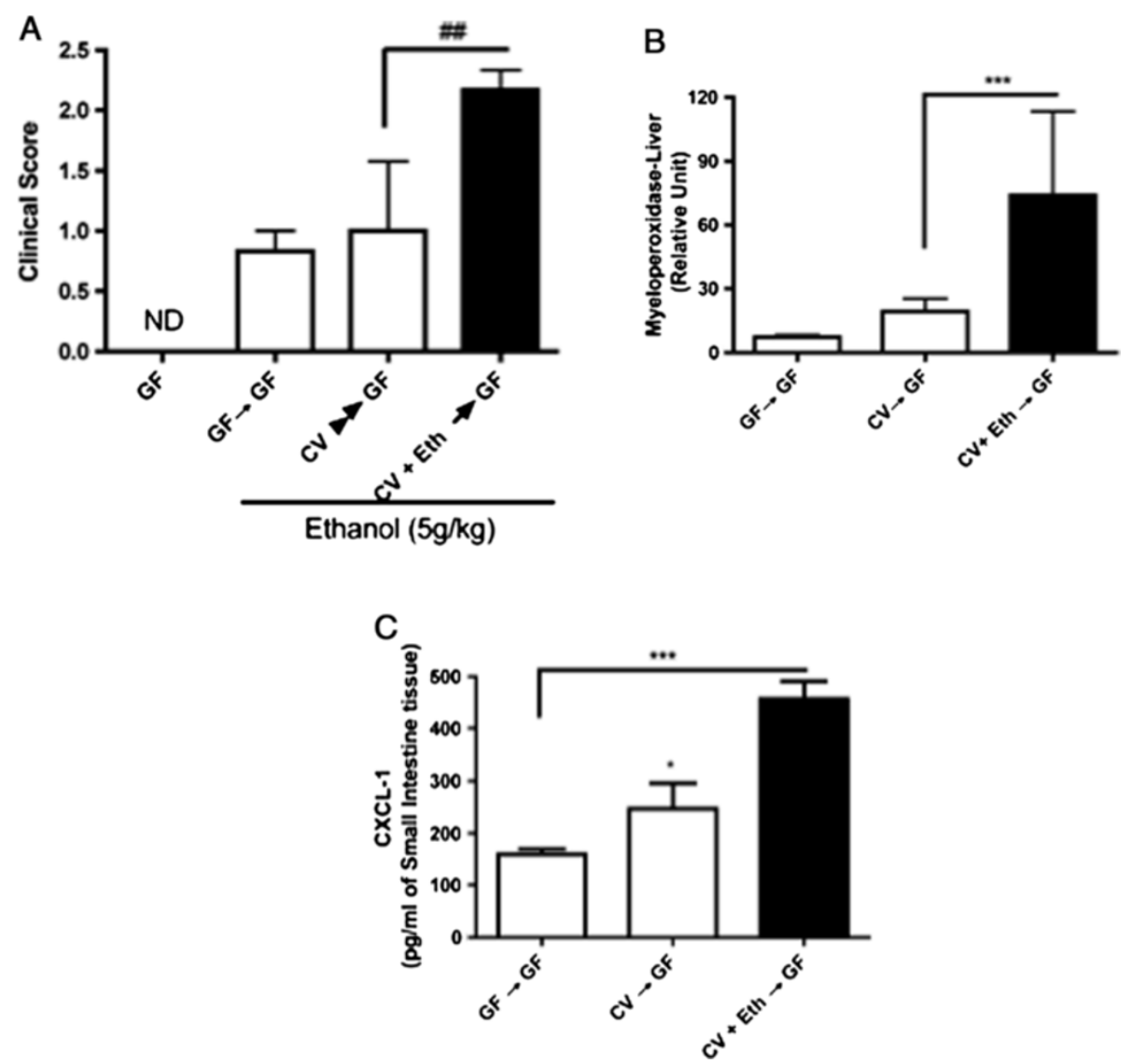

Figure 4 Influence of alcohol treatment on the clinical score and inflammatory parameters in the small intestines of conventionalized mice. Clinical score (A). The number of neutrophils that accumulated in the small intestine was measured using an MPO assay (B). Levels of the pro-inflammatory cytokine CXCL-1/KC in the small intestine $(\mathbf{C})$. The cytokine levels were measured by ELISA. The data represent the mean \pm SEM ( $n=5$-7/group). ${ }^{*} p<0.05,{ }^{* *} p<0.01$ and ${ }^{* * *} p<0.001$.

Fiber treatment reduces gut permeability and protects conventional mice from liver injury after acute alcohol consumption

As a high-fiber diet is known to promote the growth of beneficial bacteria, to control possible dysbiosis in the gut, and to protect individuals from inflammation [12,13], conventional mice were treated with a high-fiber diet to assess the possible protective effect on gut leakage after alcohol consumption. Treatment with a high-fiber diet $(\mathrm{HF}+$ Ethanol) decreased intestinal permeability after excessive alcohol intake, which was not observed in mice treated with a low-fiber diet before alcohol intake (LF + Ethanol) (Figure 5A). As IL- $1 \beta$ is known to be critical in the protection of epithelial cells [14], its levels were also measured in the small intestines of all of the groups tested (Figure 5B). The levels of this cytokine were similar in the groups receiving the LF and HF diets but decreased sharply in the LF + Ethanol group. Consumption of a high-fiber diet also restored IL-1 $\beta$ levels to normal values in the small intestines of the alcohol-fed animals. Liver score histology evaluation confirmed these data in HF +
Ethanol mice, which showed reduced microvacuole density in the liver (implying a reduction in lipid accumulation in the liver) compared with the density in the LF + Ethanol group. The latter group presented a high density of lipid microvacuoles in the liver, which is characteristic of early-stage steatosis (Figure 5C, D).

\section{Discussion and conclusions}

ALD constitutes a large proportion of liver disease worldwide. Despite extensive investigation over the past few decades, we still do not fully understand the mechanism of this disease and therefore lack an effective therapy. The results obtained in the present study, comparing germfree and conventional mice submitted to acute alcohol intake, confirm that the gut microbiota plays an important role in alcohol-induced liver injury. In particular, in the absence of intestinal microbiota, there is no inflammation and no liver disease. Moreover, alcohol-induced liver injury seems to be associated with dysbiosis of the intestinal microbiota, most likely caused by alcohol intake. Treatment with a high-fiber diet prevented this alcohol-induced 


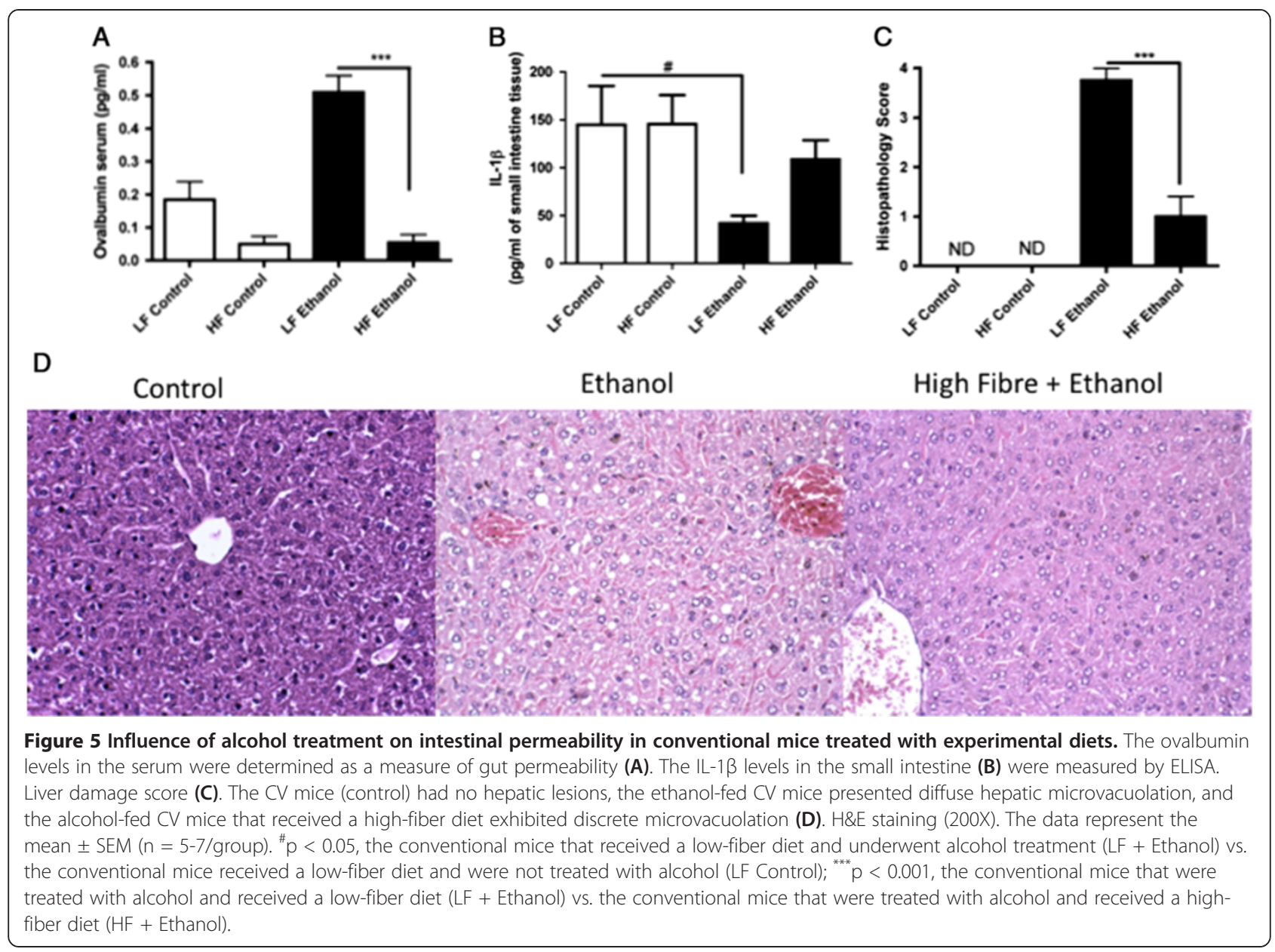

liver injury and gut leakage, providing an interesting alternative for treating the consequences of alcoholism.

Previous studies have shown the gut-liver axis is an important pathway in the development of liver injury after excessive alcohol consumption [1-3]. The two main mechanisms of gut barrier disruption involve the metabolites of alcohol, such as acetaldehyde, and a change in the composition of the gut microbiota caused by alcohol $[15,16]$. These alterations allow the passage of gut-derived endotoxins into the liver, leading to liver injury [17]. Here, we showed that in the absence of intestinal microbiota, no liver injury was observed after alcohol feeding in mice. Furthermore, these mice did not show any changes in their levels of the inflammatory cytokines/chemokines CXCL-1/KC and IL-6. These results indicate that alcohol metabolites alone are not sufficient for the development of hepatocyte pathology and that the presence of an altered microbiota and its products is also necessary. Additionally, LPS has been reported as a key bacteria-derived product that induces inflammation in alcoholic patients $[7,17]$, as it is found at high levels in the circulation of alcoholics with liver disease $[4,5,18]$. Thus, treatment with an endotoxin (LPS)-neutralizing protein significantly suppresses the alcohol-induced elevation of plasma endotoxin levels and liver injury and inhibits TNF- $\alpha$ production [19]. These studies suggest the importance of the altered gut microbiota and gut permeability in increasing LPS levels in the plasma during ethanol consumption.

The balance of gut bacteria, intestinal permeability and hepatocyte function appears to be critical for maintaining normal homeostasis of the gut-liver axis. Alcohol consumption affects the composition of the gut microbiota, induces bacterial overgrowth (Gram negative) and allows more bacterial products to travel from the intestine to the liver $[17,20]$. Dysbiosis is thus an important factor in the pathogenesis of ALD in patients with leaky intestines. Here, we show that following alcohol consumption, there is a significant alteration in the gut microbiota, which can be transferred from one animal to another, as demonstrated by the worse liver injury observed in the $\mathrm{CV}+$ Eth $\rightarrow$ GF mice compared with the CV $\rightarrow$ GF mice. This injury was characterized by increased hepatocyte pathology and higher levels of inflammatory mediators in $\mathrm{CV}+\mathrm{Eth} \rightarrow \mathrm{GF}$ mice.

The alteration in the gut microbiota consists of an increase in the total number of bacteria observed, mainly 
in the Enterobacteriaceae group, of which Escherichia coli is a representative species. Interestingly, this group of bacteria is associated with several pathological conditions in the gastrointestinal tract, such as inflammatory bowel disease [21-23]. These results align with the literature, which has described alterations in the composition of the microbiota after alcohol intake in humans and mice $[6,24]$. Interestingly, treatment with the probiotic Lactobacillus, a Firmicutes bacterium whose number is decreased in alcohol-fed mice, protects mice from alcohol-induced liver injury [6,25], thereby demonstrating the importance of restoring the composition of the normal microbiota in alcohol-induced disease.

Multiple factors likely contribute to the changes that occur in the intestinal microbiota during the development of ALD. These changes include small intestinal dysmotility [26], changes in gastric acid secretion [27], and alterations in the innate immune response in the intestine. This situation justifies attempts to restore eubiosis, which might in turn restore intestinal homeostasis [24] to treat liver disease. It is well established that diet affects the composition of the gut microbiota $[12,28,29]$, so this approach may be a way to manipulate the microbial ecosystem. Prebiotics are defined as food ingredients that specifically promote the growth of beneficial bacteria and consequently promote both homeostasis in the gut and good health [30]. Soluble fiber has certain characteristics of prebiotics (they pass intact through the small intestine and are metabolized in the colon by components of the local microbiota) but are used only by a specific group of beneficial bacteria. Nevertheless, we analyzed the effects of a high-fiber diet enriched with pectin on alcohol-derived liver disease. The results showed that the animals fed a high-fiber diet showed less liver injury and lower intestinal permeability after alcohol consumption compared with the animals that received a lowfiber diet. The beneficial effect of fiber ingestion could be due to prevention and/or reversal of the effects of alcohol on barrier integrity and/or to compensation for dysbiosis of the microbiota. The latter effect could restore the normal commensal bacteria, such as Firmicutes bacteria, which are known to produce short-chain fatty acids (SCFAs). In addition to these possibilities, another putative mechanism that may explain fiber's benefits involves an increase in IL-1 $\beta$ production in the intestine. Although IL- $1 \beta$ is classically known as a pro-inflammatory cytokine, recent studies have shown that it is also involved in epithelial repair [28]. Dietary fiber can be broken down into SCFAs, such as acetate, propionate and butyrate, by the normal intestinal microbiota to obtain energy [28,30]. These SCFAs bind to epithelial cells, inducing the activation of inflammasomes. Inflammasomes are cytoplasmic multi-protein complexes that sense microbial products and are composed of NLRs, adapter proteins, and procaspase-1, which trigger IL- $1 \beta$ and IL-18 maturation [31]. The data in the present study show that the IL-1 $\beta$ levels in the intestine were increased in animals fed a high-fiber diet and that this cytokine possibly protected these mice against alcohol-induced liver injury. These results align with those of a recent study in which increased levels of IL-1 $\beta$ were observed in the liver in association with hepatic regeneration after alcohol-induced injury [15]. Moreover, SCFAs stimulate the release of mucin, which is important for mucus secretion, providing physical protection against invasion by pathogenic bacteria and preventing an increase in gut permeability [32]. Thus, our data are the first to show that dietary fiber can preserve gut permeability after alcohol intake, providing an interesting alternative therapy for alcoholic patients.

In summary, the present study suggests that the indigenous intestinal microbiota is involved in liver injury due to high alcohol consumption. Apparently, changes in the composition of the gut microbiota (dysbiosis) induce an increase in gut permeability and subsequent trafficking of bacterial products to the liver, causing damage. In addition, the data show that ingestion of a high-fiber diet decreases gut permeability and liver injury after alcohol intake and thus could be an interesting therapy for alcoholic patients.

\section{Methods}

\section{Mice}

Eight- to ten-week-old female germ-free NIH Swiss mice were obtained from Taconic Farms (Germantown, NY, USA) and maintained in flexible plastic isolators (Standard Safety Equipment, McHenry, MD, USA) using classical gnotobiology techniques [33]. Conventional NIH Swiss mice are derived from germ-free matrices and are considered to be conventional only two generations after conventionalization. Water and a commercial autoclavable diet (Nuvilab, Nuvital, Curitiba, PR, Brazil) were sterilized by steam and administered ad libitum. For experiments, animals were maintained in micro-isolators (UNO Roestvaststaal, The Netherlands) located in a ventilated animal caging system (Alesco Ltd., Campinas, SP, Brazil) with controlled lighting ( $12 \mathrm{~h}$ light, $12 \mathrm{~h}$ dark), humidity $(60-80 \%)$ and temperature $\left(22 \pm 1^{\circ} \mathrm{C}\right)$. Experiments using germ-free mice were conducted under aseptic conditions to avoid infecting the animals [34]. All procedures complied with the standards stated in the Guide for the Care and Use of Laboratory Animals (Institute of Laboratory Animal Resources, National Academy of Sciences, Bethesda, MD, 1996) and were conducted under conditions approved by the local animal ethics committee (CETEA/UFMG, 119/2012).

\section{Experimental design}

Groups of 5-7 animals were used to separately evaluate the influences of microbiota, conventionalization and 
high-fiber treatment. For determination of the influence of microbiota, the following four groups of mice were tested: GF Control: germ-free control; GF + Ethanol: germ-free treated with alcohol; CV Control: conventional control; and CV + Ethanol: conventional treated with alcohol. For determination of the influence of conventionalization, the following four groups were tested: GF: germ-free control; GF $\rightarrow$ GF: germ-free treated with alcohol; $\mathrm{CV} \rightarrow \mathrm{GF}$ : germ-free conventionalized with intestinal contents from conventional control; and $\mathrm{CV}+$ Eth $\rightarrow$ GF: germ-free conventionalized with intestinal contents from conventional treated with alcohol. Finally, for determination of the influence of fiber, the following four groups were tested: LF: conventional fed a low-fiber diet; HF: conventional fed a high-fiber diet; LF + Ethanol: conventional fed a low-fiber diet and treated with alcohol; and HF + Ethanol: conventional fed a high-fiber diet and treated with alcohol.

\section{Alcohol treatment}

The alcohol treatment protocol included administering ethanol to the mice $(10 \% \mathrm{vol} / \mathrm{vol})$ in their drinking water for 7 days, with additional oral gavage with a higher dose of alcohol ( $5 \mathrm{mg} / \mathrm{kg})$ on day 7 . The mice were then sacrificed at different time points after the oral gavage [35].

\section{Conventionalization}

The process of conventionalization of germ-free mice with microbiota from conventional mice was performed as previously described $[33,36]$. Briefly, the intestinal contents were removed from the large intestine of germfree mice $(\mathrm{GF} \rightarrow \mathrm{GF})$, conventional mice $(\mathrm{CV} \rightarrow \mathrm{GF})$, and conventional mice that had undergone the alcohol treatment $(\mathrm{CV}+\mathrm{Eth} \rightarrow \mathrm{GF})$ and were homogenized in saline $(10 \%)$ in an anaerobic chamber (Forma Scientific Company, Marietta, OH, USA) with an atmosphere of $85 \% \mathrm{~N}_{2}, 15 \% \mathrm{H}_{2}$ and $5 \% \mathrm{CO}_{2}$. The homogenates were then administered by oral gavage to germ-free mice. Fourteen days later, these animals were subjected to the alcohol treatment protocol, as described above. To assess whether there was adequate colonization of the germ-free mice, fecal samples were cultured using a thioglycollate test [36]

\section{Fiber treatment}

To assess the effect of fiber treatment, fourteen days prior to ethanol administration and during the entire experimental period, conventional animals were supplied either with the AIN93 [37] modified as a special highfiber diet using enrichment with $10 \%$ pectin-soluble fiber (HF) or with a low-fiber diet (LF) (Additional file 2).

\section{Assessment of clinical score}

The mice were monitored for nine hours after the final high dose of ethanol was administered via oral gavage.
They were then left alone in a cage for 10 minutes to obtain fecal samples for analysis. Fecal blood was tested using Hemoccult test cards (INLAB-Diagnostica, São Paulo, Brazil). Graded scores were given as follows: $0=$ feces with a normal consistency and no blood in the fecal blood test, 1 = feces with a smooth consistency and no blood in the fecal blood test, 2 = feces with a pasty consistency and a low level of blood in the fecal blood test, and 3 = liquid feces and a high level of blood in the fecal blood test.

\section{Quantification of neutrophil accumulation}

The extent of neutrophil accumulation in the liver tissue was measured by assaying MPO activity, as previously described [11]. Briefly, liver tissue was removed and snap-frozen in liquid nitrogen. Upon thawing and processing, the tissue was assayed for MPO activity by measuring the change in the optical density at $450 \mathrm{~nm}$ using tetramethylbenzidine. The results were expressed as the neutrophil index, which denotes the activity of MPO related to casein-elicited murine peritoneal neutrophils processed in the same manner.

\section{Measurement of cytokine and chemokine concentrations in the small intestine and liver}

The levels of IL- $1 \beta$, IL- 6 , and CXCL-1/KC were measured in small intestine and liver tissue samples using commercially available antibodies according to the manufacturer's instructions (R\&D Systems, Minneapolis, MN, USA).

\section{Gut permeability assessment}

Gut permeability was evaluated in the animals by administering one dose ( $80 \mathrm{mg} / \mathrm{animal}$ ) of ovalbumin (Sigma, St. Louis, MI, USA) 90 minutes before the high dose of alcohol on day 7. Sera were then collected from these mice, and ELISAs were performed to detect the ovalbumin level in the blood. The level of ovalbumin was directly proportional to the gut permeability. FITC-dextran (MW 4000; Sigma, St. Louis, MI, USA) gavage was also used to assess gut permeability. More specifically, after 4 hours of fasting, the mice were gavaged with $500 \mathrm{mg} / \mathrm{kg}$ of FITC-dextran. The serum level of FITC-dextran was then measured in blood harvested 4 hours after administration.

\section{Total liver lipids}

For hepatic lipid measurements, $100 \mathrm{mg}$ of liver tissue was homogenized at $4^{\circ} \mathrm{C}$ in lysis buffer containing $50 \mathrm{mM}$ Tris (pH 8.0), $150 \mathrm{mM} \mathrm{NaCl}$, and 1\% Triton X-100. Lipids were extracted from the total liver homogenate using the chloroform-and-methanol method [38]. 


\section{Histological assessment}

Liver specimens were fixed in $10 \%$ neutral buffered formalin and embedded in paraffin. Histological sections $(4 \mu \mathrm{m})$ were then stained with hematoxylin and eosin (H\&E) or periodic acid-Schiff (PAS), coded, examined and graded by two independent investigators that were blind to the samples according to published criteria for magnitude analysis of steatosis [39] . Briefly, the degree of steatosis was graded $0-4$ based on the average percent of fat accumulated hepatocytes per field at $\times 200$ magnification (Grading $0=<5 \%, 1=5 \sim 25 \%, 2=26 \sim 50 \%, 3=$ $51 \sim 75 \%, 4=>75 \%)$.

\section{Statistical analysis}

Analyses were performed using the GraphPad Prism 5.3 software. The data are shown as the mean \pm SEM. Comparisons between two groups were performed using Student's $t$-test for unpaired data. Three or more group comparisons were carried out using one-way ANOVA followed by Student-Newman-Keuls multiple comparisons test. A $P$ value less than 0.05 were considered significant.

\section{Additional files}

Additional file 1: Analysis of hydric consumption among experimental groups used in the present study.

Additional file 2: Composition of the high-fiber (HF) (10\% pectin, modified AIN93M diet) and low fiber (LF) diets.

\section{Competing interests}

The authors declare that they have no competing interest.

\section{Authors' contributions}

The authors' contributions were as follows: MCCC and ATV conceived and designed the experiments. MCCC, NLQ, CM, JL, DA, SHP, CFM and ATV performed the experiments. MCCC, MMT and ATV analyzed the data. COG and GDC performed the histological analyses. FSM and JRN provided the germ-free mice. ALGB and MMT contributed reagents/materials/analysis tools. MCCC, ALGB, MMT, JRN and ATV wrote and corrected the paper. All authors read and approved the final manuscript.

\section{Acknowledgments}

The authors thank IIma Marçal (ICB/UFMG) for technical support. This work was supported by Conselho Nacional de Desenvolvimento Científico e Tecnológico (CNPq) , Coordenação de Aperfeiçoamento de Pessoal de Nível Superior (CAPES) and Fundação de Amparo e Pesquisa de Minas Gerais (FAPEMIG- APQ 01921-12). CNPq, CAPES and FAPEMIG had no role in the design, analysis or writing of this article.

\footnotetext{
Author details

1Department of Physiology and Biophysics, Institute of Biological Sciences, Universidade Federal de Minas Gerais, Belo Horizonte, Brazil. ${ }^{2}$ Department of General Biology, Institute of Biological Sciences, Universidade Federal de Minas Gerais, Belo Horizonte, Brazil. ${ }^{3}$ Department of General Pathology, Institute of Biological Sciences, Universidade Federal de Minas Gerais, Belo Horizonte, Brazil. ${ }^{4}$ Department of Microbiology, Institute of Biological Sciences, Universidade Federal de Minas Gerais, Belo Horizonte, Brazil. ${ }^{5}$ Department of Biochemistry and Immunology, Institute of Biological Sciences, Universidade Federal de Minas Gerais, Belo Horizonte, Brazil. ${ }^{6}$ Department of Pharmacology, Institute of Biomedical Sciences - ICB-1, São Paulo University, São Paulo, Brazil.
}

Received: 17 May 2014 Accepted: 2 September 2014

Published online: 16 September 2014

\section{References}

1. O'Shea RS, Dasarathy S, McCullough AJ, Practice Guideline Committee of the American Association for the Study of Liver D, Practice Parameters Committee of the American College of G: Alcoholic liver disease. Hepatology 2010, 51:307-328.

2. Purohit V: International collaboration on alcoholic liver disease and pancreatitis: opportunities. J Gastroenterol Hepatol 2006, 21(Suppl 3):S107-S108.

3. Gao B, Bataller R: Alcoholic liver disease: pathogenesis and new therapeutic targets. Gastroenterology 2011, 141:1572-1585.

4. Nanji AA, Khettry U, Sadrzadeh SM: Lactobacillus feeding reduces endotoxemia and severity of experimental alcoholic liver (disease). Exp Biol Med 1994, 205:243-247.

5. Bode C, Kugler V, Bode JC: Endotoxemia in patients with alcoholic and non-alcoholic cirrhosis and in subjects with no evidence of chronic liver disease following acute alcohol excess. J Hepatol 1987, 4:8-14.

6. Yan AW, Fouts DE, Brandl J, Starkel P, Torralba M, Schott E, Tsukamoto H, Nelson KE, Brenner DA, Schnabl B: Enteric dysbiosis associated with a mouse model of alcoholic liver disease. Hepatology 2011, 53:96-105.

7. Bhagwandeen BS, Apte M, Manwarring L, Dickeson J: Endotoxin induced hepatic necrosis in rats on an alcohol diet. J Pathol 1987, 152:47-53.

8. Ramaiah SK, Jaeschke H: Hepatic neutrophil infiltration in the pathogenesis of alcohol-induced liver injury. Toxicol Mech Methods 2007, 17:431-440.

9. Mutlu E, Keshavarzian A, Engen P, Forsyth CB, Sikaroodi M, Gillevet P: Intestinal dysbiosis: a possible mechanism of alcohol-induced endotoxemia and alcoholic steatohepatitis in rats. Alcohol Clin Exp Res 2009, 33:1836-1846.

10. Mutlu EA, Gillevet PM, Rangwala H, Sikaroodi M, Naqvi A, Engen PA, Kwasny M, Lau CK, Keshavarzian A: Colonic microbiome is altered in alcoholism. Am J Physiol Gastrointest Liver Physiol 2012, 302:G966-G978.

11. Barcelos LS, Talvani A, Teixeira AS, Cassali GD, Andrade SP, Teixeira MM: Production and in vivo effects of chemokines CXCL1-3/KC and CCL2/JE in a model of inflammatory angiogenesis in mice. Inflamm Res 2004, 53:576-584.

12. De Filippo C, Cavalieri D, Di Paola M, Ramazzotti M, Poullet JB, Massart S, Collini S, Pieraccini G, Lionetti P: Impact of diet in shaping gut microbiota revealed by a comparative study in children from Europe and rural Africa. Proc Natl Acad Sci U S A 2010, 107:14691-14696.

13. Turnbaugh PJ, Ley RE, Mahowald MA, Magrini V, Mardis ER, Gordon Jl: An obesity-associated gut microbiome with increased capacity for energy harvest. Nature 2006, 444:1027-1031.

14. Franchi L, Kamada N, Nakamura Y, Burberry A, Kuffa P, Suzuki S, Shaw MH, Kim YG, Nunez G: NLRC4-driven production of IL-1beta discriminates between pathogenic and commensal bacteria and promotes host intestinal defense. Nat Immunol 2012, 13:449-456.

15. DeSantis DA, Ko CW, Liu Y, Liu X, Hise AG, Nunez G, Croniger CM: Alcohol-induced liver injury is modulated by NIrp3 and NIrc4 inflammasomes in mice. Mediat Inflamm 2013, 2013:751374.

16. Szabo G, Bala S, Petrasek J, Gattu A: Gut-liver axis and sensing microbes. Dig Dis 2010, 28:737-744.

17. Szabo G, Bala S: Alcoholic liver disease and the gut-liver axis. World J Gastroenterol 2010, 16:1321-1329.

18. Fukui $H$, Brauner B, Bode JC, Bode C: Plasma endotoxin concentrations in patients with alcoholic and non-alcoholic liver disease: reevaluation with an improved chromogenic assay. J Hepatol 1991, 12:162-169.

19. Zhou Z, Wang L, Song Z, Lambert JC, McClain CJ, Kang YJ: A critical involvement of oxidative stress in acute alcohol-induced hepatic TNF-alpha production. Am J Pathol 2003, 163:1137-1146.

20. Wang Y, Hu Y, Chao C, Yuksel M, Colle I, Flavell RA, Ma Y, Yan H, Wen L: Role of IRAK-M in alcohol induced liver injury. PLOS One 2013, 8:e57085.

21. Bonnet M, Buc E, Sauvanet $P$, Darcha C, Dubois D, Pereira B, Dechelotte $P$, Bonnet R, Pezet D, Darfeuille-Michaud A: Colonization of the human gut by E. coli and colorectal cancer risk. Clin Cancer Res 2014, 20:859-867.

22. Chassaing B, Darfeuille-Michaud A: The commensal microbiota and enteropathogens in the pathogenesis of inflammatory bowel diseases. Gastroenterology 2011, 140:1720-1728.

23. Darfeuille-Michaud A, Boudeau J, Bulois P, Neut C, Glasser AL, Barnich N, Bringer MA, Swidsinski A, Beaugerie L, Colombel JF: High prevalence of 
adherent-invasive Escherichia coli associated with ileal mucosa in Crohn's disease. Gastroenterology 2004, 127:412-421.

24. Schnabl B, Brenner DA: Interactions Between the Intestinal Microbiome and Liver Diseases. Gastroenterology 2014, 146:1513-1524.

25. Yan AW, Schnabl B: Bacterial translocation and changes in the intestinal microbiome associated with alcoholic liver disease. World I Hepatol 2012, 4:110-118.

26. Wegener M, Schaffstein J, Dilger U, Coenen C, Wedmann B, Schmidt G: Gastrointestinal transit of solid-liquid meal in chronic alcoholics. Dig Dis Sci 1991, 36:917-923.

27. Bode C, Bode JC: Alcohol's role in gastrointestinal tract disorders. Alcohol Health Res World 1997, 21:76-83.

28. Macia L, Thorburn AN, Binge LC, Marino E, Rogers KE, Maslowski KM, Vieira AT, Kranich J, Mackay CR: Microbial influences on epithelial integrity and immune function as a basis for inflammatory diseases. Immunol Rev 2012, 245:164-176.

29. Peng X, Li S, Luo J, Wu X, Liu L: Effects of dietary fibers and their mixtures on short chain fatty acids and microbiota in mice guts. Food Funct 2013, 4:932-938.

30. Vieira AT, Teixeira MM, Martins FS: The Role of Probiotics and Prebiotics in Inducing Gut Immunity. Front Immunol 2013, 4:445.

31. Schroder K, Tschopp J: The inflammasomes. Cell 2010, 140:821-832.

32. Barcelo A, Claustre J, Moro F, Chayvialle JA, Cuber JC, Plaisancie P: Mucin secretion is modulated by luminal factors in the isolated vascularly perfused rat colon. Gut 2000, 46:218-224

33. Pleasants JE: Letter: Legislation-the solution to a dilemma. J Oral Surg 1974, 32:166.

34. Fagundes $C T$, Amaral FA, Souza AL, Vieira AT, Xu D, Liew FY, Souza DG, Teixeira MM: ST2, an IL-1R family member, attenuates inflammation and lethality after intestinal ischemia and reperfusion. J Leukoc Biol 2007, 81:492-499.

35. Bertola A, Park O, Gao B: Chronic plus binge ethanol feeding synergistically induces neutrophil infiltration and liver injury in mice: A critical role for E-selectin. Hepatology 2013, 58:1814-1823.

36. Souza DG, Vieira AT, Soares AC, Pinho V, Nicoli JR, Vieira LQ, Teixeira MM: The essential role of the intestinal microbiota in facilitating acute inflammatory responses. J Immunol 2004, 173:4137-4146.

37. Reeves PG, Nielsen FH, Fahey GC Jr: AIN-93 purified diets for laboratory rodents: final report of the American Institute of Nutrition ad hoc writing committee on the reformulation of the AIN-76A rodent diet. J Nutr 1993, 123:1939-1951.

38. Folch J, Lees M, Sloane Stanley GH: A simple method for the isolation and purification of total lipides from animal tissues. J Biol Chem 1957, 226:497-509.

39. Wang Y, Seitz HK, Wang XD: Moderate alcohol consumption aggravates high-fat diet induced steatohepatitis in rats. Alcohol Clin Exp Res 2010, 34:567-573.

doi:10.1186/s12866-014-0240-4

Cite this article as: MCC et al:: Comparing the effects of acute alcohol consumption in germ-free and conventional mice: the role of the gut microbiota. BMC Microbiology 2014 14:240.

\section{Submit your next manuscript to BioMed Central and take full advantage of:}

- Convenient online submission

- Thorough peer review

- No space constraints or color figure charges

- Immediate publication on acceptance

- Inclusion in PubMed, CAS, Scopus and Google Scholar

- Research which is freely available for redistribution 